\title{
Removing Speckle Noise in Synthetic Aperture Radar Images using Linear Filters
}

\author{
D.Suresh, S.Manimekalai, V.Nandakumar, N.Dhanalakshmi
}

\begin{abstract}
Speckle is a granular aggravation, typically demonstrated as a multiplicative noise that influences Synthetic aperture radar (SAR) pictures, just as every single cognizant picture. In the course of the most recent three decades, a few techniques have been proposed for the decrease of spot, or despeckling, in SAR pictures. The examination begins with the linear filtering, non-linear filtering, adaptive filtering, and hybrid filtering. In spite of the fact that the old style straight separating strategies have lower execution similarly, the hybridization between them beats than the as of late proposed techniques. Be that as it may, the cautious determination of such filters and their impacting request exceptionally influences the presentation of such filtering methodologies. In this paper, Hybrid filtering is proposed for SAR despeckling, which involves the improved variants of frost, median and mean filters. The presentation of the proposed framework is broke down and contrasted and the as of late SAR despeckling strategies. The outcomes demonstrate that the hybrid filters are focused as they can despeckle the SAR pictures superior to the current methods.
\end{abstract}

\section{Keywords : Speckle noise,SAR images, Despeckling,NLM}

\section{INTRODUCTION}

Synthetic Aperture Radar (SAR) imaging is more appropriate for various atmosphere conditions 1 than the optical remote detecting. Be that as it may, the serious issue in SAR imaging is the nearness of spot, a sign ward granular commotion, which debases the quality and investigation of the picture. Henceforth, it is imperative to diminish the spots from the SAR pictures before the picture examination begins, simultaneously, the picture subtleties, for example, target focuses, surfaces, shapes ought not be get upset. In the course of the most recent decades there are various research commitment on despeckling, as the new age of SAR imaging has normally gains the consideration of scientists towards this issue. The idea of despeckling calculation is firmly related with Additive White Gaussian Noise (AWGN) denoising. Notwithstanding, the noise is multiplicative in nature; the log-change is applied on the sign to change over them into added substance to fit the methodologies with additive noise filtering technique. There are three classes of despeckling calculations (I) spatial, (ii) wavelet-based, and (iii) nonlocal separating.

The spatial filtering systems take a little piece and supplant the objective pixel of the window by methods for a weighted normal. For a low-pass filter, where the loads are

Revised Manuscript Received on January 2, 2020.

Dr.D.Suresh, Assistant Professor,Department of Computer Science and Engineering,PSNA College of Engineering and Technology, Dindigul.

S.Manimekalai, Assistant Professor,Department of English, PSNA College of Engineering and Technology, Dindigul.

V.Nandakumar, Assistant Professor,Department of Computer Science and Engineering, PSNA College of Engineering and Technology, Dindigul.

Dr.N.Dhanalakshmi, Assistant Professor,Department of Computer Science and Engineering, PSNA College of Engineering and Technology, Dindigul. equivalent, diminishes the noise as well as disturbs the edges, structures and surfaces. Considering the numerous perception of a similar sign is one answer for this issue, and the loads must be evaluated dependent on measurable models of sign and noise and, the neighborhood insights of the picture. In spite of the fact that the improved spatialfilters offers better despeckling, recognizing homogeneous areas from surface or edges is a troublesome task.2223.Filtering in recurrence space is appropriate for SAR pictures as modest number of coefficients is sufficient to catch a large portion of the sign substance. It is examined in the writing that the wavelet based strategies are enough for SAR despeckling as it ensures improved execution than the spatial filters. Also, the wavelet based filters are move, pivot and scale invariant. Be that as it may, upsets in edges and the heterogeneous locales makes the SAR pictures outwardly irritating now and again. Nonlocal separating is the ongoing methodology pursues the idea of spatial filtering 31. Here the piece and the objective pixel are picked as spatial-filter does, yet the thing that matters is that the weighted normal is assessed with the comparative parts encompassing the objective pixel. Nonlocal Means (NLM) calculation demonstrated its proficiency and brought forth exceptional research. This thought is additionally created in square coordinating 3-D (BM3D)32 where just a couple of pixels with the most comparative setting are utilized for the estimation. Clearly, the piece determination is impacted by noise itself, particularly in level territories of the picture, which can be hazardously self-referential.Ingeneral, hybrid filter separating is another methodology which consistently exists in the race, likewise focused. Cautious choice of channels and their pipelining request characterizes the exhibition of the methodology. In this paper a hybrid filtering methodology with wiener, middle and mean filters to build a channel which contains all the three filters qualities and to defeat ones downside with others.

The remainder of the paper is portrayed as pursues: the accompanying area depicts the foundation of separating strategies to hybrid filter later. Segment 3 clarifies the proposed hybrid filter. Segment 4 shows the numerical outcomes and investigates the exhibition of the hybrid filterl. Area 5 finishes up the paper.

\section{EXISTING FILTERING TECHNIQUES}

Normally filters used for noise removal as follows

Edge based Adaptive Mean Filter

Local Adaptive Median Filter

Adaptive Frost Filtering.

The following table summarizes the characteristics of mean, median and Wiener filters. 


\begin{tabular}{|l|l|l|l|}
\hline Filter & $\begin{array}{l}\text { Frost } \\
\text { Filter }\end{array}$ & $\begin{array}{l}\text { Mean } \\
\text { Filter }\end{array}$ & $\begin{array}{l}\text { Median } \\
\text { Filter }\end{array}$ \\
\hline Merits & $\begin{array}{l}\text { Linear } \\
\text { filter }\end{array}$ & Non-linear & $\begin{array}{l}\text { Non- } \\
\text { linear }\end{array}$ \\
\cline { 2 - 4 } & $\begin{array}{l}\text { Additive } \\
\text { Noise }\end{array}$ & & $\begin{array}{l}\text { Salt \& } \\
\text { Pepper, } \\
\text { Speckle } \\
\text { Noise }\end{array}$ \\
\hline & $\begin{array}{l}\text { Adaptive } \\
\text { filtering }\end{array}$ & Smoothing & $\begin{array}{l}\text { Preserve } \\
\text { Sharp } \\
\text { edges }\end{array}$ \\
\hline \multirow{7}{*}{ Demerits } & $\begin{array}{l}\text { Not } \\
\text { suitable } \\
\text { for } \\
\text { speckle } \\
\text { Noise }\end{array}$ & $\begin{array}{l}\text { Can't } \\
\text { preserve } \\
\text { edges }\end{array}$ & $\begin{array}{l}\text { Not } \\
\text { Adaptive }\end{array}$ \\
& & \\
\hline
\end{tabular}

\section{PROPOSED FMM HYBRID FILTERING}

There is another approach of filtering called hybrid filter, used to blend more than one filters together to acquire the upside of all. 20 proposed a calculation utilizing Hybrid request insights channel HMM (Hybrid Mean Median) is proposed, which is a blend of mean and middle filters to smother speckle noise in SAR shading pictures. The exhibition measurements shows that the proposed filter performs better as far as PSNR and MSE. 4 Proposed an improved nearby insights channel for sifting the speckle noise from the SAR pictures. The proposed filter is a blend of mean and hybrid median filters, utilizing a novel $7 \times 7$ filtering format. The presentation of the proposed filter is tried against the standard Hybrid Median filters for which the assessed qualities show better performs as far as PSNR and SSI. Additionally, this filter has a novelistic property of expelling noise, nearby edge maintenance and subtleties conservation. 23proposed an unaided change recognition system by utilizing k-implies grouping on the consolidated contrast picture. This technique utilizes the neighborhood consistency of the distinction picture in the wake of utilizing the mean filter and the edge data conservation of the distinction picture in the wake of utilizing the median filter for better contrast picture portrayal.

The primary thought of this paper is to hybrid these filters to take every one of their points of interest while the negative marks of each filter could be settled by the benefits of another. For the hybridization the improved variants of mean, median and frost filters, for example, Edge based Adaptive Mean (EAM) filter 33 Local Adaptive Median (LAM) filter 32 and the Adaptive Frost (AF) filter 31 are utilized. Henceforth the hybridization filter is Linear, Adaptive, Preserve the edges, appropriate for speckle noise and furthermore smoothen the pictures. In this paper, the above talked about filters are hybrid together for SAR despeckling. Figure 1 represents the proposed Frost-MedianMean (FMM) filter system.

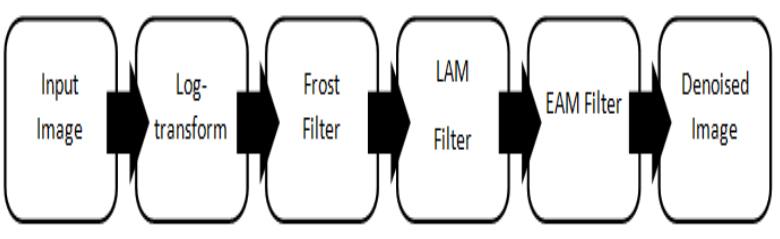

Figure 1. The Proposed Hybrid Filter Framework The steps involved in the proposed algorithm is

Initially the image is applied with $\log$ transformation to convert the multiplicative noise into additive, now the signal is suitable for the first step of filtering where the Adaptive Frost filter is applied.

In the next step, Locally Adaptive Median(LAM) filtering is applied to preserve the image details like edges and textures. The limitations of median filter like nonadaptiveness are resolved as it is applied after frost filtering.

Finally for the overall smoothing, the EAM filtering is applied to avoid the ridges caused by previous filtering at homogeneous regions.

\section{EXPERIMENTS \& RESULTS}

The proposed framework is studied with both real and simulated SAR images. And their performance is studied with three different performance measures like PSNR (Peak Signal-to-Noise Ratio, Signal to Clutter Ratio, and Edge Preservation Index). The following figure shows the results of the hybrid filter from simulated and real images.

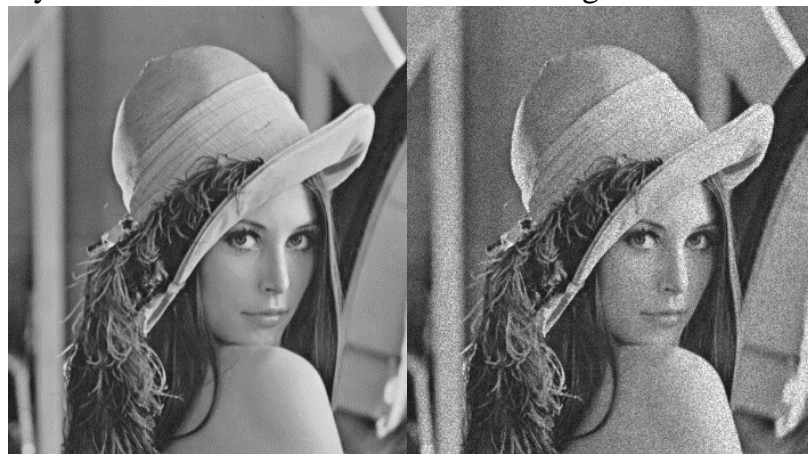

(a) ( b)

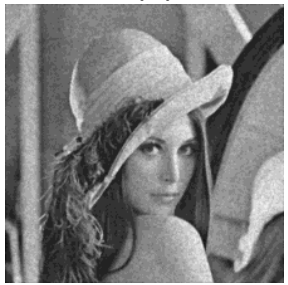

(c)

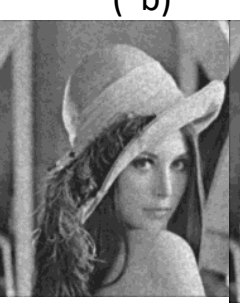

(d)

(e)
PS 27.8832
28.2999
29.1152
4.1407
4.1843
4.2187
R
0.6587
0.8043

Figure 2.Despeckling Results of Hybrid Filter with Simulated SAR Image (a) Original Image, (b) Noisy Image, (c) Adaptive Frost Filter Result, (d) AF+LAM Filter Result, and (e) AF+LAM+EAM Filter Result 


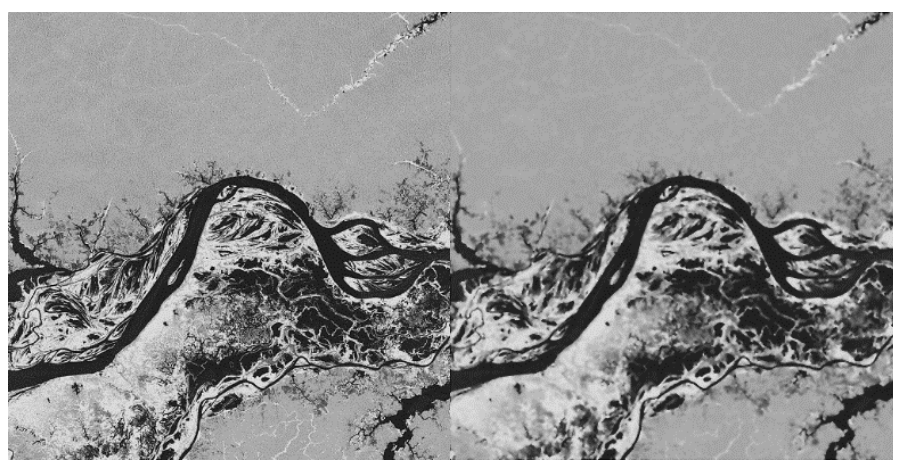

(a)

(b)

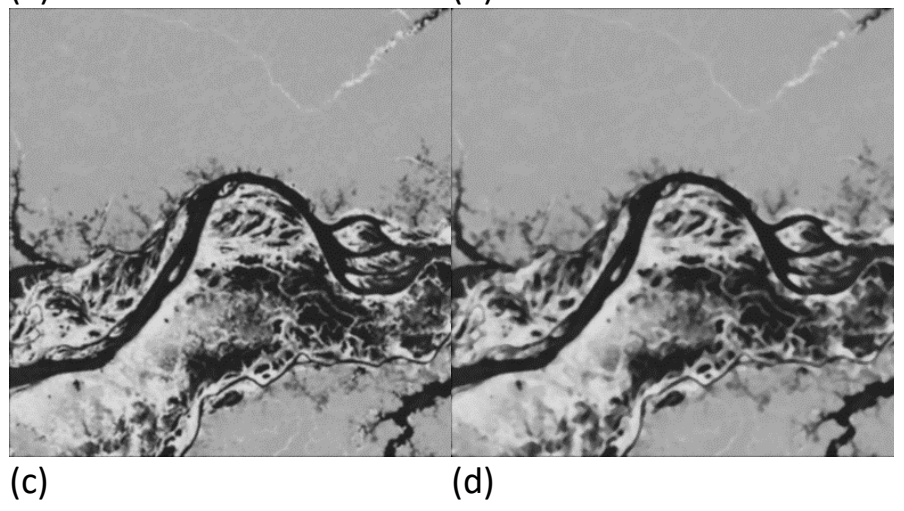

Figure 3.Despeckling Results of Hybrid Filter with

Real SAR Image (a) Original Image, (b)Adaptive Frost

Filter Result, (c) AF+LAM Filter Result, and (d) AF+LAM+EAMFilter Result

Further the proposed FMM hybrid filter is compared with the four other existing despeckling approaches BM3D,7 SWW 21, Frost Filter 1, Mean-Median4. Table 1 quantifies performance comparison of despeckling methods. The results indicate that the FMM hybrid filter outperforms the existing approaches.

Table 1. Performance Comparison of Despeckling

\begin{tabular}{|l|l|l|l|}
\hline Filtering Methods & PSNR & SCR & EPI \\
\hline FMM & 29.7254 & 4.7021 & 0.9171 \\
\hline BM3D & 28.2131 & 4.2151 & 0.8122 \\
\hline SWW & 28.1187 & 4.1201 & 0.8005 \\
\hline Frost Filter & 25.5647 & 3.8751 & 0.7517 \\
\hline Mean-Median & 24.3157 & 3.8111 & 0.7721 \\
\hline
\end{tabular}

\section{CONCLUSIONS}

Synthetic Aperture Radar (SAR) imaging is influenced by Speckle noise in nature, which makes its understanding harder. Various filtering strategies like spatial filtering, frequency-domain filtering, non-local filtering and hybrid filtering approaches are displayed in the writing. Among these the non-local mean filtering beats ongoing days, and hybrid filters produces comparative outcomes as NLM filters. This paper proposes a hybrid filter for SAR despeckling, where the Frost, Median and Mean filters are assembled for developing a novel hybridization called FMM (Frost-Median-Mean) filtersl. The SAR pictures are applied with these filters individually sequentially and the outcome in broke down and contrasted and the other denoising strategies. The outcomes demonstrate that the proposed FMM filters has unrivaled execution than the current filters in the spatial, frequency, hybrid just as non-local domains.

\section{REFERENCES}

1. Jain, A. K. (1989). Fundamentals of digital image processing. Prentice-Hall, Inc..

2. J. Trinder, Edge-detection-based filter for SAR noise reduction, IJRS, vol. 33, no. 7, pp. 2296- 2320, Apr 2012.

3. Lee J.-SPolarimetric Radar Imaging fro $\mathrm{m}$ Basics to Applications , pp. 143-152 (New York: CRC Press).

\section{AUTHORS PROFILE}

Dr.D.Suresh, Assistant Professor,Department of Computer Science and Engineering,PSNA College of Engineering and Technology, Dindigul.

S.Manimekalai, Assistant Professor,Department of English, PSNA College of Engineering and Technology, Dindigul.

V.Nandakumar, Assistant Professor,Department of Computer Science and Engineering, PSNA College of Engineering and Technology, Dindigul.

Dr.N.Dhanalakshmi, Assistant Professor,Department of Computer Science and Engineering, PSNA College of Engineering and Technology, Dindigul. 